\title{
ON THE ORIGIN OF METALLICITY IN LYMAN-ALPHA FOREST SYSTEMS
}

\author{
M. CHIBA \\ National Astronomical Observatory, Mitaka, Tokyo 181, JAPAN \\ AND \\ B. B. NATH \\ Raman Research Institute, Bangalore-560080, INDIA
}

\section{Introduction}

Several recent observations indicate that even the low $\mathrm{H}$ I column density Ly $\alpha$ absorption lines may not be primordial, as they were previously thought to be. High-resolution observations using the Keck telescope (Cowie et al. 1995; Tytler et al 1995.; Songaila \& Cowie 1996) have revealed that a large fraction of $\operatorname{Ly} \alpha$ absorption lines with $\mathrm{H}$ I column density as low as $N_{H I} \sim 10^{14} \mathrm{~cm}^{-2}$ at redshift $z \sim 3$ are associated with metal lines (C IV). This fraction, hereafter denoted as $f_{m}$, is estimated as $0.5-0.75$. On the other hand, there is an indication that at low $z$ a large fraction of Ly $\alpha$ absorption lines are directly associated with halos of visible galaxies. This fraction is hereafter denoted as $f_{g}$. Lanzetta et al. (1995, hereafter LBTW) claimed $f_{g} \sim 0.6$ at $z \lesssim 1$ (see also Bowen, Pettini \& Boyle 1997).

In the hierarchical model of structure formation, there is a continuum of halos of various masses and velocity dispersions. In this contribution, we consider the hypothesis that the Ly $\alpha$ absorption lines are produced in two populations of halos in this continuum - in 'minihalos' of small velocity dispersions that have never cooled and formed stars, and in large galactic halos with stars and metals. Based on this two-population model for Ly $\alpha$ absorbers, together with chemical history of galactic halos, we investigate whether or not $f_{m}$ is understood in terms of $f_{g}$ by the relationship $f_{m} \simeq f_{g}$. If $f_{m}$ is larger than $f_{g}$, it may be concluded that the intergalactic medium (IGM) experienced a global enrichment at higher $z$. 

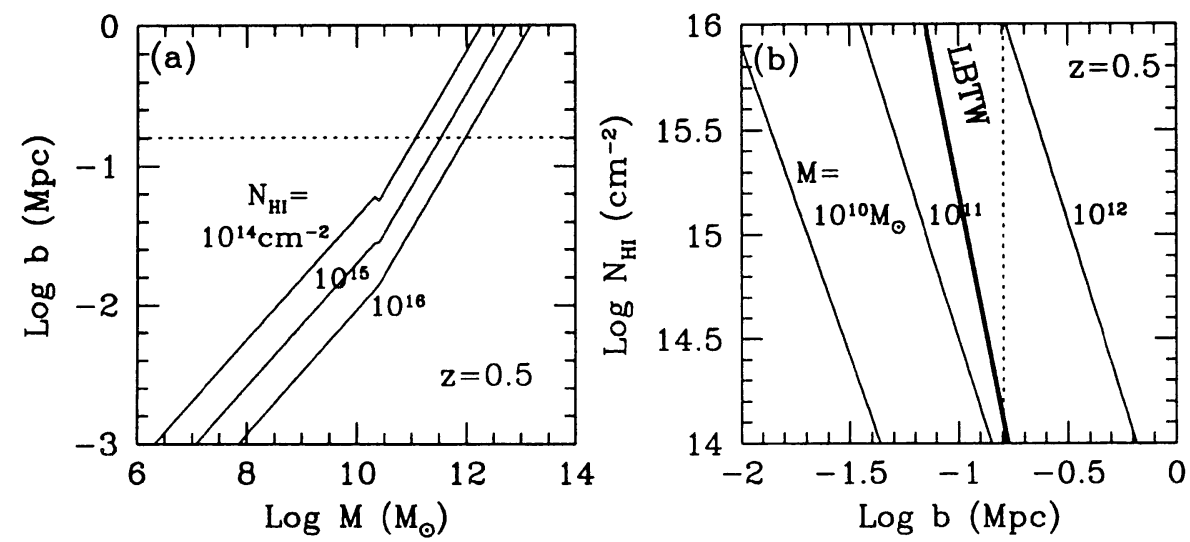

Figure 1. (a) The impact parameter $b(\mathrm{Mpc})$ of a sightline as a function of the total mass of a halo $M\left(M_{\odot}\right)$ to produce an H I column density $N_{H I}$ at $z=0.5$. (b) The H I column density $N_{H I}\left(\mathrm{~cm}^{-2}\right)$ against the impact parameter $b(\mathrm{Mpc})$.

\section{Two-population model for $\operatorname{Ly} \alpha$ absorbers}

We assume that the density profile of both minihalos and galactic halos is represented by that of a softened isothermal sphere, which is characterized by the circular velocity $V_{c}$. We define minihalos as halos in which baryonic gas is entirely photoionized by the external UV background and the gas is stably confined in the gravitational field of the dark matter (Rees 1986). Minihalos are bounded by two scales of $V_{c}, V_{1}<V_{c}<V_{2}$, where the lower limit $V_{1}$ is set by the sound velocity of the photoionized IGM, which is $\sim 15\left(T_{I G M} / 10^{4} \mathrm{~K}\right)^{0.5} \mathrm{~km} \mathrm{~s}^{-1}$. The upper limit $V_{2}$ is taken as $\sim 55 \mathrm{~km} \mathrm{~s}^{-1}$, following the argument by Thoul and Weinberg (1996) on galaxy formation under the UV background. We will call the halos with $V_{2}<V_{c}<V_{3}$ galactic halos. Our results below are not sensitive to the upper limit $V_{3}$, and we will use a value of $250 \mathrm{~km} \mathrm{~s}^{-1}$. In this case, Ly $\alpha$ absorbers in galactic halos are assumed to be confined with ambient hot halo gas (Mo 1994).

Figure 1(a). shows the relation between the impact parameter $b$ and the total mass of the system $M$ when $N_{H I}$ is given. It is seen that the H I column densities as low as $10^{14} \mathrm{~cm}^{-2}$ can be provided by either the low-mass minihalos with $b \lesssim 40 \mathrm{kpc}$ or by the high-mass galactic halos with larger $b$. Figure 1(b) shows $N_{H I}$ vs. $b$ for a given total mass. The dotted line denotes the observed size of gaseous envelopes $\sim 160 \mathrm{~h}^{-1} \mathrm{kpc}$ in luminous galaxies that give rise to $\operatorname{Ly} \alpha$ absorption lines at $z \lesssim 1$ (LBTW). The thick line indicates the observed correlation between $N_{H I}$ and $b$ obtained by LBTW. These results suggest that the current model reproduces the observational results in a reasonable manner. 

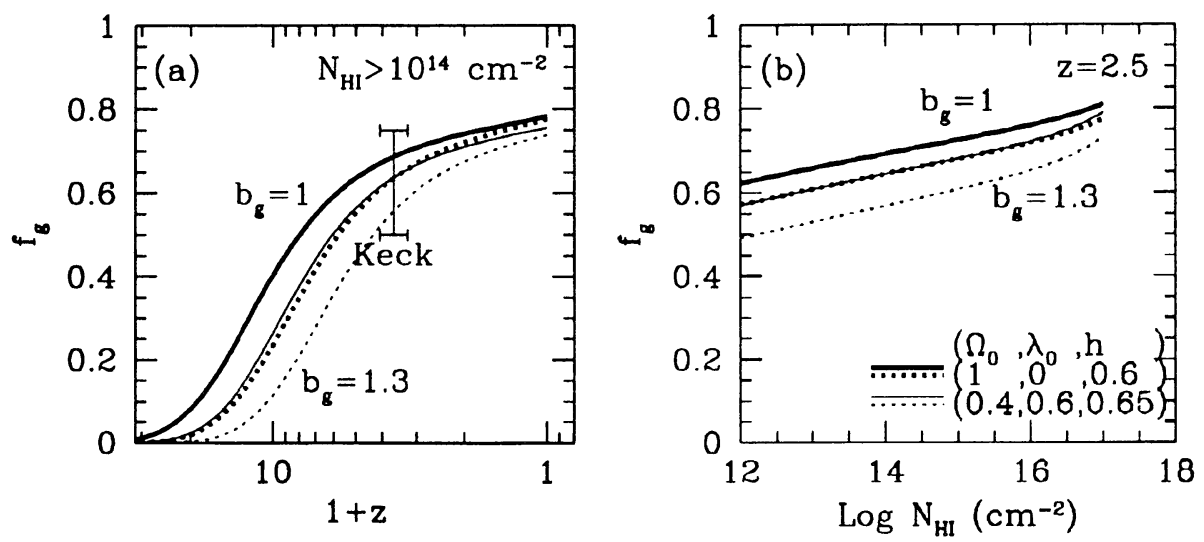

Figure 2. (a) The redshift evolution of the fraction of $\operatorname{Ly} \alpha$ absorption lines associated with galactic halos, $f_{g}$, in the range of $55 \leq V_{c} \leq 250 \mathrm{~km} \mathrm{~s}^{-1}$ (while $15 \leq V_{c} \leq 55 \mathrm{~km} \mathrm{~s}^{-1}$ for minihalos), with $N_{H I}>10^{14} \mathrm{~cm}^{-2}$. The error bar denotes the Keck results for the fraction of Ly $\alpha$ lines associated with CIV metal lines. (b) The dependence of $f_{g}$ on $N_{H I}$ at $z=2.5$.

\section{The number of $\operatorname{Ly} \alpha$ absorption lines}

Based on the Press-Schechter formalism for deriving the mass function of collapsed halos, we calculate the number of $\operatorname{Ly} \alpha$ absorption lines per unit redshift $d N / d z$ in the universe, which is specified by cosmological parameters $\left(H_{0}, \Omega_{0}, \lambda_{0}\right)$. The power spectrum of density perturbations is taken from the CDM, with a bias parameter $b_{g}$ for normalization. It is found that the value of $d N / d z$ decreases with decreasing $z$ as the hierarchical mergings proceed. Detailed comparison with observations suggests that the model reproduces the observed number of Ly $\alpha$ lines, as long as $V_{1}$ is about $15 \mathrm{~km} \mathrm{~s}^{-1}$ (see Chiba \& Nath 1997 for details).

We then evaluate the fraction of Ly $\alpha$ absorbers associated with galactic halos, $f_{g}$, by calculating the number of absorbers in the range $V_{2}<V_{c}<V_{3}$ for galactic halos. Figure 2(a) shows the redshift evolution of $f_{g}$ for lines with $N_{H I}>10^{14} \mathrm{~cm}^{-2}$, where we assume $V_{2}=55 \mathrm{~km} \mathrm{~s}^{-1}$. It is found that at high $z(z \gtrsim 10)$, most of the Ly $\alpha$ lines are provided by minihalos that collapsed early in the CDM power spectrum. The fraction $f_{g}$ then increases with time as a result of the formation of massive halos via hierarchical merging. It is worth noting that the predicted value of $f_{g}$ at $z \sim 2.5$ are reasonably in agreement with the reported value of $f_{m}$ for metal lines. We also obtain the gradual increase of $f_{g}$ with increasing $N_{H I}$ [Figure 2(b)], which is to be confirmed from observations. 


\section{Metallicity of $\operatorname{Ly} \alpha$ absorbers}

Several recent observations have also measured the metal abundances of Ly $\alpha$ lines at high $z$ (Cowie et al. 1995; Womble et al. 1996). The presence of heavy elements implies that interstellar gas confined in dark halos has been processed by star formation and subsequent chemical evolution. Here we take the following simplified approach to evaluating the metallicity of Ly $\alpha$ lines associated with galactic halos. First, absorbers confined with gaseous halos are assumed to be enriched as a result of formation of metalpoor halo stars and/or globular clusters. According to observations and models of these population II objects in the Galaxy (Schuster \& Nissen 1989; Pagel 1989), the rate of metal enrichment is of the order of $10^{-3}$ $\mathrm{Gyr}^{-1}$ from the possible age-metallicity relationship, and the process of enrichment lasted for a few Gyrs from the observed spread in ages. Second, star formation from gas components is assumed to start when background dark halos collapse. The latter epochs are evaluated from typical epochs of collapse for $1 \sigma$ density fluctuations.

Figure 3 shows the evolution of the mean abundance for absorption lines with $N_{H I}>10^{14} \mathrm{~cm}^{-2}$ (solid and dotted lines). It is found that the model reasonably reproduces the Keck results of $[\mathrm{C} / \mathrm{H}]=-2.5 \sim-2$ at $z \sim 2.5$. We assume $\log \langle Z\rangle / Z_{\odot}=-1$ as an upper limit for abundance of absorption lines, since the subsequent formation of halo stars may be inhibited as a result of gas accretion onto the disk component. Also shown in the figure with filled circles are the recent findings of Lu et al. (1996) for the abundances $([\mathrm{Fe} / \mathrm{H}])$ of damped Ly $\alpha$ systems observed with Keck. Intriguingly, the median values of abundances in such systems are well reproduced by the current models. This may support the hypothesis, first proposed by Lanzetta, Wolfe \& Turnshek (1995), that damped Ly $\alpha$ systems could represent a spheroidal component of galaxies.

\section{Concluding remarks}

We have shown that a fraction of Ly $\alpha$ forest lines with associated metal lines can be understood in the framework of hierarchical structure formation model, assuming that halos with circular velocity $15 \lesssim V_{c} \lesssim 55 \mathrm{~km} \mathrm{~s}^{-1}$ do not produce stars and heavy elements. The model predicts that the fraction of Ly $\alpha$ lines with associated metal lines increases slowly with the H I column density. We have further used the age-metallicity relation of Galactic halo stars to predict the evolution of the average metallicity of the associated metal line systems. In particular, we find that the average metallicity at $z \sim 2$ should be about $1 / 100$ th of solar.

As an observational test for this model, we propose to determine the firm limits on the fraction $f_{m}$ of Ly $\alpha$ lines associated with metal lines, 


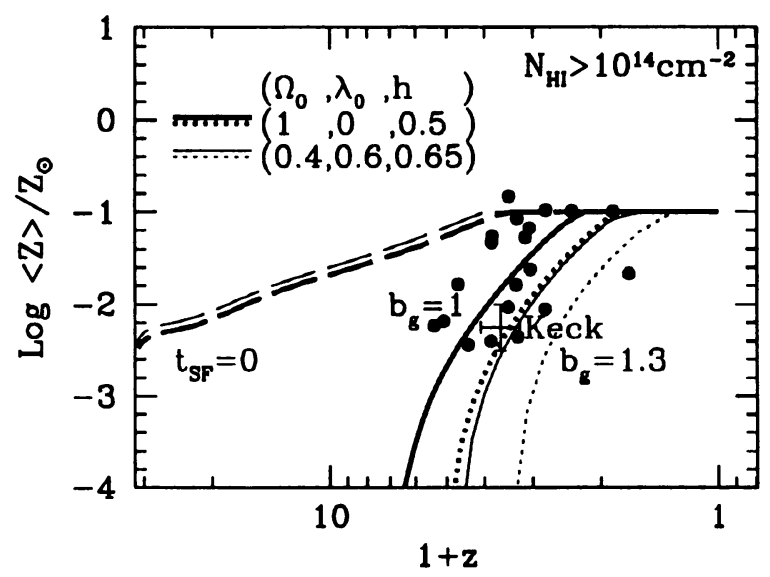

Figure 3. The redshift evolution of the average metallicity $\langle Z\rangle$ of absorption lines based on the model described in the text. For reference, the metallicity evolution if the star formation commences from the beginning of the universe, $t_{S F}=0$, are shown as dashed lines.

dependence of $f_{m}$ on $z$ and $N_{H I}$, and dependence of metallicity in Ly $\alpha$ absorbers on $z$.

\section{References}

Bowen, D.V., Pettini, M., \& Boyle, B.J. 1997, MNRAS, in press (astro-ph/9707306)

Chiba, M. \& Nath, B.B. 1997, ApJ, 483, 638

Cowie, L.L., Songaila, A., Kim, T.S., \& Hu, E.M. 1995, AJ, 109, 1522

Lanzetta, K.M., Wolfe, A.M., \& Turnshek, D.A. 1995, ApJ, 440, 435

Lanzetta, K.M., Bowen, D.B., Tytler, D., \& Webb, J.K. 1995, ApJ, 442, 538 (LBTW)

Lu, L., Sargent, W.L.W., Barlow, T.A., Churchill, C.W., \& Vogt, S.S. 1996, ApJS, 107, 475

Mo, H.J. 1994, MNRAS, 269, L49

Pagel, B.E.J. 1989, Rev. Mexicana Astron. Astrofis., 18, 161

Rees, M.J. 1986, MNRAS, 218, 25P

Schuster, W.J. \& Nissen, P.E. 1989, A\&A, 222, 69

Songaila, A., \& Cowie, L.L. 1996, AJ, 112, 335

Thoul, A.A. \& Weinberg, D.H. 1996, ApJ, 465, 608

Tytler, D., Fan, X.-M., Burles, S., Cottrell, L., Davis, C., Kirkman, D., \& Zuo, L. 1995,, in QSO Absorption Lines, ed. G. Meylan (Berlin: Springer-Verlag), p. 289

Womble, D.S, Sargent, W.L.W., \& Lyons, R.S. 1996, in Cold gas at High Redshift, eds. M. Bremer, H. Rottgering, P. van der Werf, C. Carilli (Kluwer), in press 